\title{
A Newly Built rAAV-SaCas9 Genome Editing System Enables Muscle- Directed Gene Editing to Improve Muscular Atrophy
}

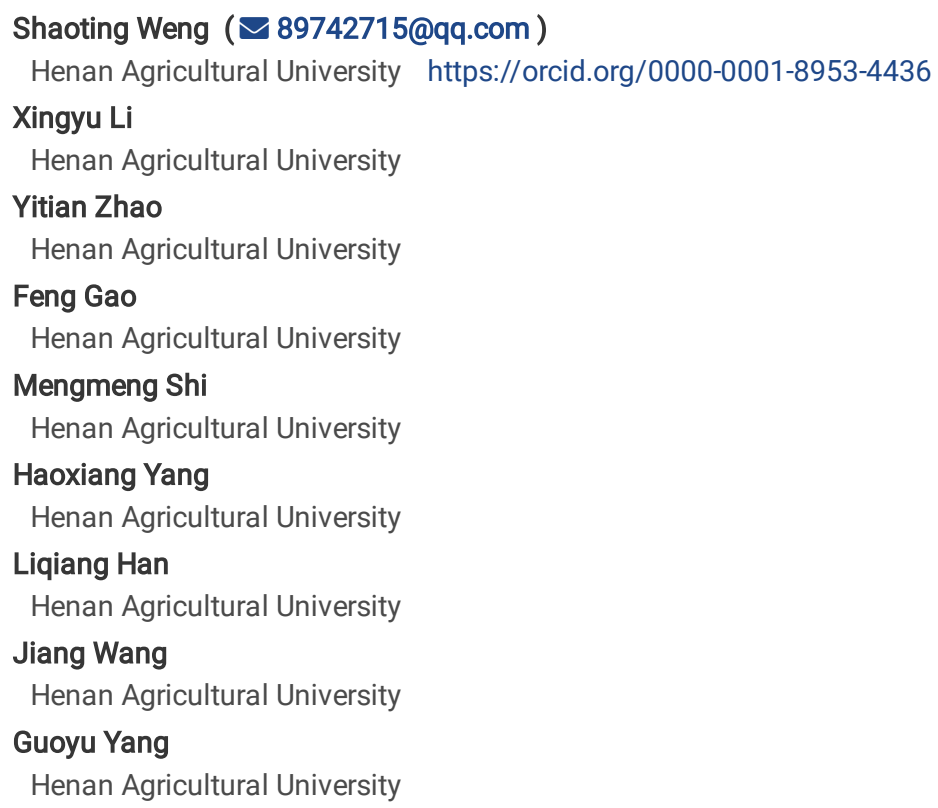

\section{Research}

Keywords: Genome editing, rAAV-SaCas9, Myostatin, Intramuscular injection, Muscular atrophy

Posted Date: May 22nd, 2020

DOI: https://doi.org/10.21203/rs.3.rs-29591/v1

License: (c) (1) This work is licensed under a Creative Commons Attribution 4.0 International License. Read Full License 


\section{Abstract \\ Background}

At present, genome editing at specific sites in vivo is affected by many factors, including the choice of vector, the efficiency of editing proteins and the influence of the internal environment. These factors make gene editing ineffective and even have adverse effects.

\section{Methods}

Here, we report a single rAAV containing SaCas 9 and guide RNAs under the control of subtle EF1a and tRNA promoters. The capacity of rAAV was compressed, and we inserted the sequence of the green fluorescent protein eGFP into rAAV. The efficiency of rAAV gene editing in vivo and in vitro was analyzed by time point and virus titer. In addition, we used the rAAV9-SaCas9 system to knock out the myostatin gene in the thigh muscles of muscleatrophic mice.

\section{Results}

We demonstrated that the gene editing elements regulated by the rAAV-SaCas 9 system can be expressed. By increasing the amount of rAAV and the reaction time, the editing efficiency of myostatin and the expression level of eGFP protein can be improved in vitro and vivo. Furthermore, We demonstrated that muscle cells were improved by knockout partial myostatin gene in a mouse model of muscular dystrophy.

\section{Conclusions}

The rAAV-SaCas9 system can be expressed in a stable and long-term manner. The system has substantial therapeutic potential in treating muscular atrophy.

\section{Background}

The CRISPR/Cas system has become a commonly used technology in biomolecular research and biomolecular therapies[1, 2]. This system can be used to create genetically modified animals[3,4], and it provides a way to resist viral invasion[5]. Moreover, genome-wide screening can be performed by using the programmable properties of CRISPR/Cas9[6, 7]. In addition, CRISPR/Cas9 has been used to genetically modify potential clinical treatments for diseases[8-10]. However, genome editing at specific sites in vivo is affected by many factors, including the choice of vector, the efficiency of editing proteins and the influence of the internal environment.

In vivo delivery of CRISPR/Cas9 has been achieved through hydrodynamic delivery or viral transduction, including with adenoviral or adeno-associated virus (AAV) vectors. In clinical settings, AAV as the vector for Cas9 provides a promising gene therapy method[11]. In particular, this technology can greatly benefit muscle atrophy treatment by enabling selective manipulation of gene expression in specific muscle regions[12, 13]. However, many of the exciting tools developed from CRISPR-SpCas9 technology are too bulky to meet the genome packaging limits of AAV ( 4.85 kb including both ITRs). One way to overcome this technical hurdle is to take advantage of smaller orthologs of Cas 9 derived from different prokaryotic species. Three Cas9 nucleases that have been recognized to be effective have similar properties to those of Streptococcus pyogenes Cas9 (SpCas9), but are markedly smaller. Notably, SpCas9 is $4.3 \mathrm{~kb}$, and it scarcely fits into the AAV genome when coupled with essential gene regulatory elements. However, Streptococcus thermophilus Cas9 (St1Cas9), Neisseria meningitidis Cas9 (NmCas9), and Staphylococcus aureus Cas9 (SaCas9) are all $1 \mathrm{~kb}$ shorter than SpCas9[14-16]. Thus, with the discovery of these smaller Cas9 nucleases, AAVs can now be engineered with either of these smaller Cas 9 genes and gRNA expression cassettes to create AAV-based CRISPR/Cas9. This technical hurdle can also be overcome by optimizing the promoter. Mefferd and coworkers have recently reported that expression of sgRNAs can be driven by small tRNA promoters $(\sim 70$ bp) with sizes approximately half that of an sgRNA-expressing cassette with a U6 promoter[17] for efficient delivery of CRISPR materials into cells. Tabebordbar and coworkers have reconstructed a new vector AAV-SaCas9 in which expression of SaCas9 is driven by EF1a-short promoters[13]. Other researchers have built the pX601miniCMV-SaCas9-U6-sgRNA vector, in which expression of SaCas9 is driven by a miniCMV promoter, which is only $39 \mathrm{bp}$.

The efficiency of expression and the smaller sizes of two or more components of a single rAAV system would be necessary to perform experiments on the system's genome-editing capabilities in aspects, including rAAV tissue tropism, and efficient precise targeting and persistent performance of organizations[18]. For example, AAV9 has strong tropism in muscle tissue, and AAV9-Cas9 gene editing can reduce the harm caused by off-target editing and prevent other tissue mutations.

Myostatin (Mstn, also known as growth differentiation factor 8 ), is a member of the transforming growth factor $\beta$ (TGF-b) signaling protein superfamily and a key regulator of muscle mass in vertebrates. Its expression inhibits the proliferation and differentiation of muscle cells. Myostatin signaling dysfunction promotes muscle growth, and myostatin-null animals have a characteristic supermuscle phenotype [19, 20]. Unsurprisingly, in muscular dystrophy diseases, including sarcopenia, muscular dystrophy, and cancer-related cachexia, controlling myostatin signaling has become an attractive prospect for increasing functional muscle mass [21-25]. The generation of effective antibodies or inhibitors targeting myostatin signals to promote 
muscle growth remains clinically challenging. Many myostatin binding antibodies, designed to treat amyotrophic disease by inhibiting myostatin signaling, have failed in primary clinical phase II trials (bimagrumab by Novartis and PINTA 745 by Atara; Novartis, 2016; Atara Bio, 2015). Similarly, an ActRIIB receptor-Fc fusion (ACE-031 by Acceleron) was withdrawn from phase II trials because of safety concerns [22]. Currently, no myostatin inhibitors are approved for clinical use, primarily because the cross-reactivity of soluble antagonists with structure-related TGF-b superfamily growth factors inhibits the transmission of mature myostatin signals[26]. However, researchers are increasingly exploring treatments for muscular dystrophy by knocking out Mstn gene expression, which has been found to be a simple and rapid way to improve muscle traits[23, 27, 28].

Here, we built a smaller rAAV-SaCas9 gene editing system, which generated EF1a-driven SaCas9 and tRNA-driven sgRNAs. We then inserted the eGFP sequence into the rAAV-SaCas9 and demonstrated the expression effects of eGFP and SaCas9 gene editing in vivo and in vitro. Furthermore, we demonstrated the efficacy of the system in mice with muscle wasting. This study provides a reference for clinical treatment applications.

\section{Materials And Methods}

\section{Construction of SaCas9 and sgRNA plasmids.}

All plasmids were constructed with standard recombinant DNA cloning techniques. pX601-CMV:SaCas9-U6:sgRNA (pX601-AAV-CMV::NLS-SaCas9NLS-3xHA-bGHpA;U6::Bsal-sgRNA) was preserved in our laboratory. tRNA $_{\mathrm{GLN}}$ and sgRNA scaffold sequences were generated by Sangon (Shanghai, China) (GGTTCCATGGTGTAATGGTTAGCACTCTGGACTCTGAATCCAGCGATCCGA

\section{GTTCAAATCTCGGTGGAACCT-}

GAAACACCGGAGACCACGGCAGGTCTCAGTTTTAGTACTCTGGAAACAGAATCTACTAAAACAAGGCAAAATGCCGTGTTTATCTCGTCAACTTGTTGGCGAGA) [17] and, after amplification with the primers tRNA GLN -F: 5'-AGGCATGCTGGGGAGGTACCGGTTCCATGGTGTAATGGTT-3' Scaf-ITR-R:5'-

CTAGGGGTTCCTGCGGCCGCAAAAATCTCGCCAACAAGTTG-3', were ligated into pX601-CMV:SaCas9-U6:sgRNA, which was digested with Kpnl and Notl, with a ClonExpress ${ }^{\circledR}$ II One Step Cloning kit (Vazyme Biotech, China). The constructed vector was then transformed into DH5a competent cells, and transformants containing pX601-CMV:SaCas9-tRNA:sgRNA were identified by sequencing. After subculturing, DNA was extracted. The EF1a promoter was amplified from the pLentiCRISPR V2 vector with the primers EF1a-F: 5'-CCTGCGGCCTCTAGACTCGAGGTGGGCAGAGCGCACATCGC-3' and EF1a-R: 5-TGGGGCCATGGTGGCACCGGTCCTGTGTTCTGGCGGCAAAC-3'. Then, pX601-CMV:SaCas9-tRNA:sgRNA, which was digested between the $\mathrm{Xhol}$ and Agel sites immediately before the SaCas9 gene, and the promoter were cloned with a ClonExpress $\AA$ II One Step Cloning kit. Vector transformation, identification and plasmid extraction were performed as described above to obtain pX601-EF1a:SaCas9-tRNA:sgRNA. The P2A-eGFP gene was amplified from th-P2A-eGFP donor with primers P2A-eGFP-F: 5'-CCGGCCAGGCAAAAAAGAAAAAGGCTACTAATTTCTCCT-3' and P2A-eGFP-R: 5'-ATCTGGAACATCGTATGGGTCTTGTACAGCTCGTC. Then, pX601-EF1a:SaCas9-tRNA:sgRNA was digested with BamH, and the P2A-eGFP gene was cloned with a ClonExpress ${ }^{\circledR}$ II One Step Cloning kit. Vector transformation, identification and plasmid extraction were performed as described above to obtain pX601-EF1a:SaCas9-eGFP-tRNA:sgRNA. The proposed structures of the vector are shown in Fig. 1A. The complementary oligonucleotides sgMstn1, sgMstn2 and sgMstn3 were annealed to form double-stranded inserts and were ligated into the Bbsl digested plasmid. Finally, the plasmids pX601-EF1a:SaCas9-eGFP-tRNA:sgMstn1, pX601-EF1a:SaCas9-eGFP-tRNA:sgMstn2 and pX601-EF1a:SaCas9-eGFP-tRNA:sgMstn3 (denoted psgMstn1, psgMstn2 and psgMstn3) were constructed.

\section{Cell culture and transfection.}

$\mathrm{NIH} / 3 \mathrm{~T} 3$ cells were cultured in Dulbecco's modified Eagle's medium (DMEM) supplemented with 10\% fetal bovine serum (FBS) and were transfected with polyethylenimine (PEI). Briefly, $5 \times 10^{5} \mathrm{NIH} / 3 \mathrm{~T} 3$ cells were cultured to $50-60 \%$ confluence in six-well plates and transfected with psgMstn1/ psgMstn2/ psgMstn3 and $1 \times \mathrm{PEI}$, and the medium was replaced with DMEM ( $3 \% \mathrm{FBS})$ after $8 \mathrm{~h}$. After $72 \mathrm{~h}$, the cells were subjected to genomic DNA extraction. All cells were cultured at $37^{\circ} \mathrm{C}$ and $5 \% \mathrm{CO}_{2}$.

\section{AAV vectors for the CRISPR/Cas9 system.}

Procedures were carried out as previously described[29]. For the production of rAAV-DJ/8, rAAV9 viruses, HEK293T cells were seeded into 20 dishes (100 $10 \mathrm{~mm} ; 5 \times 10^{6}$ cells per dish). After $24 \mathrm{~h}$, the cells were triple-transfected with pX601/psgMstn1, pAAV-DJ/8-RC pAAV9-RC and pHelper according to the instructions provided with the $1 \times \mathrm{PEI}$ transfection reagent. After $72 \mathrm{~h}$, the cells were harvested by scraping into medium, centrifuged at $1000 \cdot \mathrm{g}$ for $10 \mathrm{~min}$ and resuspended in $1 \mathrm{~mL}$ of $1 \times$ phosphate-buffered saline. The cell suspension was subjected to three freeze-thaw cycles at $\varangle 80^{\circ} \mathrm{C}$ and at $37^{\circ} \mathrm{C}$. After fast centrifugation and filtration, the cell debris was cleared. The viral solution was concentrated with PEG 8000 and purified on a cesium chloride density gradient column. After two rounds of ultracentrifugation, the high-density viruses were separated and extracted, and run through dialysis bags for desalting[30]. The titers of the purified rAAV-DJ/8, rAAV9 viruses were determined with a RT-PCR-based method described previously[31]. pX601 was diluted from $10^{9}$ copies/ $\mu \mathrm{L}$ to $10^{3}$ copies/ $\mu \mathrm{L}$ as the standard solution. The primers were ITR-QPCR-F: $5 \rrbracket-$ CGGCCTCAGTGAGCGA-3》 and ITR-QPCR-R: 5囚-AGGAACCCCTAGTGATG-3囚. The resulting viruses were designated AAV-DJ/8-EF1a:SaCas9-eGFPtRNA:sgMstn1, AAV9-EF1a:SaCas9-eGFP-tRNA: sgMstn1 and AAV-DJ/8-CN, AAV9-CN (denoted rAAV-DJ/8-sgMstn1, rAAV9-sgMstn1 and rAAV-DJ/8-CN, rAAV9-CN, respectively).

rAAV-DJ/8 transduction in vitro. 
Adeno-associated virus (AAV) serotypes differ broadly in transduction efficacy and tissue tropism. Recombinant AAV-DJ vectors provide superior in vitro transduction efficacy to that of any other wild type serotype. Efficient transduction is beneficial for gene editing in the SaCas9 system. After digestion and resuspension of $\mathrm{C} 2 \mathrm{C} 12$ cells in logarithmic growth phase, the cells were cultured in DMEM supplemented with $10 \%$ FBS in 12 -well plates at a seeding density of $1 \times 10^{5}$ and grown overnight. The cells were cultured to $50-60 \%$ confluence and infected with $\sim 2 \times 10^{9} \mathrm{vg}$ and $1 \times 10^{10} \mathrm{vg}$ rAAVDJ/8-sgMstn1, and control cells were infected with $\sim 2 \times 10^{9} \mathrm{vg}$ and $1 \times 10^{10} \mathrm{vg} \mathrm{rAAV-DJ/8-CN}$. Then, the medium was replaced with DMEM (3\% FBS) after $24 \mathrm{~h}$. Fluorescence was determined, and DNA and protein were extracted at 96 and $120 \mathrm{~h}$.

\section{Mice.}

120 C57BL/10 male mice in SPF grade (6 weeks of age) were purchased from the Center of Experimental Animal of Guangdong province (Guangzhou, China) and maintained in a specific-pathogen-free animal facility according to the Guide for the Care and use of Laboratory Animals and the related ethical regulations at Henan Agricultural University. Then, these mice were fed to the age of 8 weeks and were healthy without any abnormalities.

\section{rAAV9 transduction in vivo.}

Compared with AAV-DJ, AAV9 is more addicted in muscle tissue; it is conducive to gene editing of muscle cells and is often applied in muscle tissue. In addition, the packaging of different types of AAV allows for broad applicability of the psgMstn1 plasmid. $60 \mathrm{C} 57 \mathrm{BL} / 10 \mathrm{male}$ mice were divided into four groups, and rAAV9-sgMstn1 and rAAV9-CN were injected into male C57BL/10 mice in two doses. Three points on the thigh muscle in the left thigh in the treatment group were injected with $\sim 1 \times 10^{10} \mathrm{vg} / 1 \times 10^{11} \mathrm{vg}$ of $\mathrm{rAAV} 9-\mathrm{sgMstn} 1 \mathrm{in} 100 \mu \mathrm{L}$ of phosphate-buffered saline $(30 \mu \mathrm{L} /$ point, $\mathrm{N}=15)$, and the control group were treated similarly with $\sim 1 \times 10^{10} \mathrm{vg} / 1 \times 10^{11} \mathrm{vg}$ of rAAV9-CN in $100 \mu \mathrm{L}$ of phosphate-buffered saline (30 $\mu \mathrm{L} /$ point, $\left.\mathrm{N}=15\right)$. After 6,8 and 10 weeks, the expression of the tissue fluorescence was determined, and the thigh muscles were collected for genomic DNA extraction, western blotting.

\section{Construction of mouse model of muscular atrophy and rAAV9 transduction.}

A mouse model of muscular atrophy was constructed as previously described[32]. $40 \mathrm{C} 57 \mathrm{BL} / 10$ male mice were divided into two groups: a DEX treatment group, which received DEX treatment once every other day for 14 days (DEX, Decadron, $0.5 \mathrm{mg} / \mathrm{kg}$ of body weight, i.p., $\mathrm{N}=30$ ), and a control group, which received saline during the same period $(\mathrm{N}=10)$. DEX and saline injections were simultaneously given each day (9:00-10:00 a.m.). Then, 20 C57BL/10 male mice with significant weight changes were selected from the DEX treatment group, and $\sim 1 \times 10^{11} \mathrm{vg}$ of rAAV9-sgMstn 1 or rAAV9$\mathrm{CN}$ was injected into the thigh muscle in muscular atrophy model mice $(\mathrm{N}=10)$. Control group mice were injected with saline. After 8 weeks, the thigh muscles were collected for genomic DNA extraction, western blotting and tissue slice experiments. Body weight was measured daily in each week of the experiment.

\section{T7 endonuclease 1 (T7E1) cleavage assay and targeted deep-sequencing analysis.}

Genomic DNA was extracted with a Tissue and Cell Culture DNA Midi kit (TianGen, Beijing, China) according to the manufacturer's instructions. The purified genomic DNA was used as a template to amplify a fragment of the Mstn gene with the following specific primers: MSTN-Test1-F: 5】-

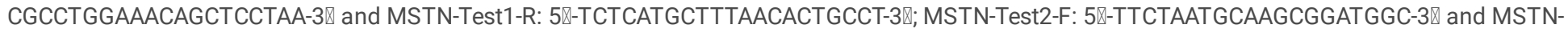

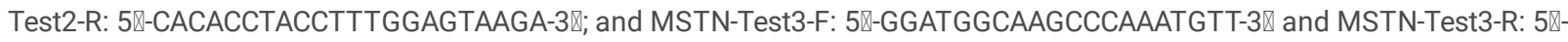

ACACACCTACCTTTGGAGTAAG-3囚. The fragment sizes amplified by these primer sets were 521 bp, 548 bp and 537 bp, respectively. The PCR products were digested with T7 endonuclease 1 (NEB, Boston, USA) and resolved with 1.5\% agarose gel electrophoresis. The primers used for tracking of insertions and deletions (indels) by decomposition (TIDE) were the same as the MSTN-Test-F/R primers. Genomic DNA (100 ng) was used for PCR amplification with a High Fidelity 2. PCR Master Mix (NEB). For TIDE analysis, 300 ng of PCR product was purified with a QIAquick PCR Purification Kit (Qiagen, Hilden, Mannheim, Germany) and sent for Sanger sequencing with the forward primer MSTN-Test-F. Indel values were obtained with the TIDE web tool (https://tide.deskgen.com/) as described previously. Targeted deep-sequencing analysis was performed for C2C12 cells and gDNA from mouse muscle with a PCR amplification approach. Briefly, the off-target locus was identified through the website http://www.rgenome.net/casoffinder/. On-target or off-target locus-specific primers (Table 1) were used to amplify the editing site with Phusion High Fidelity DNA Polymerase. The resultant amplicons were separated on a 1.0\% agarose gel. The bands of 150 bp were extracted with a SanPrep DNA Gel Extraction kit. Then, targeted deep-sequencing of DNA products was performed by GENEWIZ Inc. In brief, the libraries were sequenced on the Illumina HiSeq platform (Illumina, Santiago, USA) in paired-end mode with a read length of $150 \mathrm{bp}$. Primary analysis was performed with built-in software, HiSeq Control Software (HCS), RTA 2.3 plus, and demultiplexing was performed with bcl2fastq 2.17. Finally, the raw data of the targeted sequencing were analyzed by bioinformatics analysts at GENEWIZ Inc. The resulting indel frequencies, sizes and distributions were then plotted with GraphPad Prism.

\section{Protein analysis.}

Protein extracts were prepared on ice by homogenization of pieces of frozen tissues or cell pellets in $500 \mu \mathrm{L}$ of RIPA lysis buffer (50 mM Tris-HCl, pH 8.0, $150 \mathrm{mM} \mathrm{NaCl}, 1 \%$ Triton X-100, $1 \%$ sodium deoxycholate, $0.1 \%$ SDS and $2 \mathrm{mM} \mathrm{MgCl}_{2}$ ) supplemented with 1:100 protease inhibitor solution (Roche, Basel, Switzerland) by passage through a syringe. With each loose- and tight-fitting piston, the samples received 30 strokes and were then centrifuged at $1000 \cdot \mathrm{g}$ for $5 \mathrm{~min}$ at $4^{\circ} \mathrm{C}$ to remove debris. The supernatant (whole cell lysate) was collected. For the isolation of membrane fractions, the supernatant was further centrifuged at $13200 \cdot \mathrm{g}$ for $20 \mathrm{~min}$ at $4{ }^{\circ} \mathrm{C}$, and the pellet was resuspended $(2 \mu \mathrm{L} / \mathrm{mg}$ tissue) in sample buffer $(2.7 \mathrm{M}$ urea, 
3.3\% SDS and 0.167 M Tris, pH 6.7). The protein concentration was estimated with a BCA assay. For protein detection, $30 \mu \mathrm{g}$ of sample was separated with $10 \%$ SDS-PAGE and then transferred to a polyvinylidene fluoride membrane. After incubation in $5 \%$ nonfat milk for $1 \mathrm{~h}$, the membrane was incubated with rabbit polyclonal anti-MSTN/GAPDH antibody (1:1000, Bioss Antibodies, China, catalogue number: bs-23012R/bs-2188R) overnight at $4{ }^{\circ} \mathrm{C}$. Then membranes were incubated with horseradish peroxidase-conjugated goat anti-rabbit (1:2000, Bioss Antibodies, catalogue number: bs0295G) antibodies for $1 \mathrm{~h}$ at room temperature. The target proteins were detected with Luminata ${ }^{\text {tw }}$ Crescendo immunoblotting HRP Substrate (Millipore, USA).

\section{Histology and immunofluorescence analyses.}

The quadriceps muscles were fixed in para-formaldehyde and embedded in paraffin for further histopathological investigations. Formalin-fixed quadriceps muscles sections were stained to investigate the density and diameter of muscle fibers by hematoxylin and eosin according to a standard protocol[33]. After the staining procedure, the slides were scanned with a microscope. At least five fields at 200 magnification were randomly selected from each section in each group for imaging. The number of fibers was counted in each field, then converted to the number of fibers per mm ${ }^{2}$. Each section was analyzed to calculate the area of individual muscle fibers $\left(\mathrm{mm}^{2}\right)$, by selecting the area of every muscle fiber ( $\left.\mathrm{mm} \mathrm{m}^{2}\right)$. All data were obtained and analyzed with Image Pro Plus 6.0 software. For immunostaining, the paraffin sections of muscle tissue were dewaxed as previously described[34], placed in a solution of sodium citrate at $100{ }^{\circ} \mathrm{C}$ for 10 minutes and then soaked in hydrogen peroxide. Cross-section samples were immunostained with mouse monoclonal anti-SaCas9 primary antibody (1:200, Epigentek, catalogue number: A-9001) and goat anti-mouse IgG-FITC (1:500, Abcam, catalogue number: ab6785) secondary antibody, and DAPI for nuclei. Muscle sections were imaged with standard fluorescence microscopy.

\section{Muscle weights of mice.}

The experimental C57BL/10 male mice were weighed on electronic scales. The body weights of the mice were recorded from weeks 1 to 8 . For the measurement of muscle weight, quadriceps and adductor muscles from the left side in the experimental mice were dissected 2 months later, and the average weight was used for each muscle. Ten mice were weighed per group.

\section{Statistical analyses.}

Data are expressed as the mean \pm standard error of the mean. Unpaired Student's t-test was used to analyze differences between two groups, and oneway ANOVA analysis of variance with Bonferroni's post-test was used for multiple group comparisons with Prism 6 (GraphPad). * P values less than 0.05 and greater than 0.01 were considered significant. ** $\mathrm{P}$ value less than 0.01 was considered to be of greater significance.

\section{Results}

\section{SaCas9/CRISPR system exhibits efficient genome editing in vitro.}

In our experiment, we focused on the design of a compact CRISPR/Cas9 system suitable for AAV delivery. We constructed a viral plasmid with gene editing efficiency. The CRISPR/Cas9 system plasmid contains an EF1a promoter controlling expression of the SaCas9 coding region fused to a selfcleaving P2A sequence attached to an eGFP sequence, and it contains a constitutively expressing sgRNA expression cassette controlled by a tRNA promoter. The P2A sequence enables the SaCas9 and the eGFP domain to yield two independent proteins from the same cistron, and the translated proteins from the two genes retain their functions. This approach can be used to indirectly understand the transfection ability of recombinant AAV and the gene editing effect mediated by SaCas9 protein through the expression level of GFP protein (Fig. 1A).

To examine the genome editing capabilities of our system, we transfected NIH/3T3 cells with the pX601-:EF1a:SaCas9-eGFP-tRNA:sgRNA plasmids in the treatment groups. The sgRNAs were designed to target the mouse Mstn locus (sgMstn1/ sgMstn2/ sgMstn3); the control groups were transfected by pX601 plasmids with an empty sgRNA (Fig. 1B). We chose to target the Mstn locus because it is a well-studied gene known for its important role in muscle of overgrowth and is important in research on some amyotrophic lateral diseases. The three sgRNAs are located at the N-terminal of the first and second exon of Mstn gene, and these sites play an important role in the synthesis of MSTN precursor proteins. At $72 \mathrm{~h}$ post transfection, the cells were observed and collected by centrifugation. The expression of eGFP from the transfected plasmid psgMstn1/psgMstn2/psgMstn3 was confirmed by cellular immunofluorescence in NIH/3T3 cells (Fig. 1C). A flow cytometry experiment was performed on NIH/3T3 cells transfected with the psgMstn1/psgMstn2/psgMstn3 plasmids. The results confirmed expression of the recombinant vectors, at an expression efficiency of $46.40 \%$, $43.81 \%$ and $42.42 \%$, respectively (Fig. 1D). Genomic DNA was isolated, and the 521 bp, 548 bp and 537 bp regions from the sgMstn1/ sgMstn2/ sgMstn3 loci were PCR amplified and examined for SaCas9-mediated editing with a T7 endonuclease digestion identification method. With this assay, if no editing occurred, a single band was visible on the gel. The results were as shown in the control groups. When editing did occur, the DNA was cut at the location of the editing, thus resulting in two smaller bands of 181 and 340 bp in length for sgMstn $1 ; 185$ and 352 bp in length for sgMstn2; and 122 and 415 bp in length for sgMstn3 (Fig. 1E). For TIDE analysis, 300 ng of PCR product was sent for Sanger sequencing with the forward primer MSTN-Test1/ 2/ 3-F. Compared with the results in the control group transfected with plasmid pX601, the sequencing results of psgMstn1/psgMstn2/psgMstn3 plasmids showed that the crest of the edited cells at the knockout site was not a single, more disorderly (Fig. 1F). Indel values were obtained with the TIDE web tool (https://tide.deskgen.com/). Cells that received the pX601 vectors did not exhibit editing of the MSTN locus. However, cells transfected with psgMstn1/psgMstn2/psgMstn3 plasmids displayed an average of $31.43 \%, 18.14 \%$ and $27.94 \%$ editing, respectively (Fig. $1 \mathrm{G}$ ). Genome editing percentages were averaged from three independent samples per group. From the above data, we determined that all three plasmids had gene editing 
ability. Compared with that of sgMstn2 and sgMstn3, the gene editing efficiency of sgMstn1 was higher, and the knock-off efficiency was lower. Therefore, sgMstn1 was selected as the first choice for in vivo and in vitro targeting of packaged rAAV vectors.

The rAAV-DJ/8-SaCas9 system efficiently targets the Mstn site in C2C12 cells. First, we built rAAV-DJ/8-sgMstn1, pseudotyped as DJ/8 serotype (Fig. 2A). Next, we tested the rAAV-DJ/8-sgMstn1 infectivity with two doses in C2C12 cells, as well as the knockout effect on MSTN protein in the cells. To do so, we infected C2C12 cells with $\sim 2 \times 10^{9} \mathrm{vg} / 1 \times 10^{10} \mathrm{vg}$ of rAAV-DJ/8-sgMstn 1 in the treatment groups. The control groups comprised C2C12 cells infected with $\sim 2 \times 10^{9} \mathrm{vg} / 1 \times 10^{10} \mathrm{vg}$ of rAAV-DJ/8-CN. All cells were observed and harvested 96 and $120 \mathrm{~h}$ later and processed as described above. The expression of eGFP in the treatment groups was confirmed by cellular immunofluorescence in C2C12 cells. No eGFP expression was observed in the control groups (Fig. 2A). A flow cytometry experiment was performed on C2C12 cells infected with the rAAV-DJ/8-sgMstn1 to confirm expression of the transgene. Their editing efficiency for low and high doses, respectively, was $4.36 \%$ and $6.21 \%$ at $96 \mathrm{~h}, 17.31 \%$ and $24.84 \%$ at $120 \mathrm{~h}$ (Fig. 2B). The eGFP expression efficiency of the high dose group was higher than that of the low dose group in vitro. Genomic DNA was isolated from a $521 \mathrm{bp}$ region from the sgMstn1 locus, PCR amplified and examined for SaCas9-mediated editing with a T7 endonuclease digestion identification method. The results indicated two smaller bands 181 and 340 bp in length for sgMstn1 (Fig. 2C). In TIDE analysis, low and high doses rAAV-DJ/8sgMstn1 resulted in an average of $8.42 \%$ and $11.94 \%$ editing at $96 \mathrm{~h}$, and $16.75 \%$ and $21.88 \%$ editing at $120 \mathrm{~h}$ (Fig. 2D). Cells that received rAAV-DJ/8$\mathrm{CN}$ did not exhibit editing of the MSTN locus. Genome editing percentages were averaged from three independent samples per group. Compared with the control groups, the treatment groups infected with low or high doses rAAV-DJ/8-sgMstn1 from 96 to $120 \mathrm{~h}$ showed a gradual decrease in MSTN protein levels (Fig. 2E). A stacked histogram showing the percentage distribution of MSTN expression at different time points in C2C12 cells indicated $80.6 \%$ and $71.5 \%$ at $96 \mathrm{~h}$, and $69.4 \%$ and $62 \%$ at $120 \mathrm{~h}$. Data represent the means \pm SD from three technical replicates (Fig. $2 \mathrm{~F}$ ). Efficient editing and MSTN protein reduction indicated the success of the delivery and activity of rAAV-DJ/8-sgMstn1 at the Mstn locus, and demonstrated that gene editing of the cells with high doses of the virus was more effective.

The rAAV9-SaCas9 system efficiently targets the Mstn site in mouse thigh muscle. We sought to assess whether our rAAV9-SaCas9 system could be used for steady expression in vivo. In these experiments, we produced rAAV9-sgMstn1, pseudotyped as 9 serotype. In the treatment groups, rAAV9sgMstn 1 were infected at low and high doses into the left thigh muscle in C57BL/10 male mice, each at a titer of $\sim 1 \times 10^{10} \mathrm{vg} / 1 \times 10^{11} \mathrm{vg}$. At 6,8 and 10 weeks later, the mice were sacrificed, and eGFP fluorescence in their bodies was detected with an in vivo imaging system. The control groups were infected with rAAV9-CN. We found that the fluorescence intensity of eGFP protein increased with time, and the fluorescence intensity of the high-dose rAAV9-sgMstn1 group was significantly higher than that of the low-dose group. (Fig. 3A). Genomic DNA from mouse muscle was isolated, and a 521 bp region from the sgMstn 1 locus was PCR amplified and examined for SaCas9-mediated editing with a T7 endonuclease digestion identification method. The results revealed two smaller bands 181 and $340 \mathrm{bp}$ in length for sgMstn 1 in the treatment groups, which were absent in the control groups. (Fig. 3B). For TIDE analysis, low dose rAAV9-sgMstn1 displayed an average of $6.31 \%, 13.11 \%$ and $18.8 \%$ editing, and high doses of rAAV9sgMstn 1 displayed an average of $9.29 \%, 17.59 \%$ and $21.68 \%$ editing at 6,8 and 10 weeks, respectively (Fig. 3C). Muscle from mice that received the rAAV9-CN did not exhibit editing of the Mstn locus. The genome editing percentages were averaged from three independent samples per group. Compared with the control group muscles, in the muscle infected with low or high doses of rAAV9-sgMstn1, MSTN protein levels were gradually reduced at 6,8 and 10 weeks (Fig. 3D). A stacked histogram showing the percentage distribution of MSTN expression at different time points in muscle after infection with the viruses indicated $85.5 \%, 73.9 \%$ and $65.7 \%$ at low doses, and $75.4 \%, 67.2 \%$ and $56.6 \%$ at high doses, at 6,8 and 10 weeks, respectively. The data represent means \pm SD from three technical replicates (Fig. 3E). Efficient editing and MSTN protein reduction indicated the success of the delivery and activity of rAAV9-sgMstn1 at the Mstn locus in mice. In addition, the results confirmed that gene editing was more efficient with high viral doses in vivo.

\section{The rAAV9-SaCas9 system effectively edited genes and improved muscle mass in muscle-atrophic mice.}

In our final experiment, we sought to test our single rAAV9-SaCas9 system in mice with muscle atrophy. We tested rAAV9-sgMstn1 in C57BL/10 male mice with muscle atrophy that showed listlessness and weight loss, and muscle cells shrink and die at the cellular level. Efficient editing and MSTN protein reduction were considered to indicate successful delivery and activity of rAAV9-sgMstn1 at the Mstn locus. Mstn deficiency led to skeletal muscle proliferation in mice. Compared with the mice injected with rAAV9-CN, in mice injected with rAAV9-sgMstn1, muscle was substantially added in the left thigh. Wild type mice were used as a negative control (Fig. 4A). There was no substantial difference in body weight between the groups injected with rAAV9-sgMstn 1 and rAAV9-CN at the beginning of the experiment, whereas at the end of the experiment, especially in the last 3 weeks after injection, an increase was observed, owing to increased weights following injection with rAAV9-sgMstn1 (P<0.01) (Fig. 4B). In addition, we clearly found the weight change of quadriceps and adductor at the injection site. Treatment with rAAV9-sgMstn1 compared with the rAAV9-CN significantly increased the quadriceps weight $(\mathrm{P}<0.01)$ and adductor weight $(\mathrm{P}<0.01)$ (Fig. $4 \mathrm{C}$ and $\mathrm{D})$. These results revealed muscle weight increases in the Mstn knockout muscle. Our negative control was wild type mice, which had a steady level of muscle growth and received saline. To elucidate the successful gene editing in mice muscles, we examined the expression of SaCas9 protein in rAAV9-sgMstn1 in muscle cells. Immunostaining revealed green fluorescence in the muscle sections of mice infected with rAAV9-sgMstn1 (Fig. 4E). Hematoxylin and eosin staining of the rAAV9-sgMstn1-treated, rAAV9-CN-treated and saline-treated groups were performed, and the transverse sections of the muscle fibers showed different density distributions. The rAAV9-CN-treated group had significantly more lytic and atrophic cells than the rAAV9-sgMstn1-treated and the saline-treated groups. And the fiber areas and numbers clearly differed in size $(20 \mu \mathrm{m})$ when the transverse sections of the muscle fibers were enlarged (Fig. 4F). According to analysis of the quadriceps muscle sections, we observed that the average area of muscle fiber cells was significantly greater in the rAAV9-sgMstn1-treated group than in those from the rAAV9-CN treated (Fig. $4 G)(0.01<P<0.05)$. By analyzing the number of muscle fibers per unit area, we found that the number of

Page 6/15 
muscle fibers treated with rAAV9-sgMstn1 increased (Fig. 4H) $(0.01<\mathrm{P}<0.05)$. The results revealed that the number of muscle fibers increased, and muscles enlarged, with decreased MSTN expression in muscle tissues.

\section{SaCas9 is highly specific in vivo.}

The possibility of off-target edits is a major concern in therapeutic CRISPR/Cas9 genome editing. Some researchers have found that SaCas9 is a naturally highly occurring genome editing platform in mice[35] [36]. Using targeted deep-sequencing of mouse cell DNA products obtained by GENEWIZ Inc., we screened for off-target sites in the mouse genome to determine whether SaCas9 maintained its minimal off-targeting profile in mice cells. $\mathrm{NIH} / 3 \mathrm{~T} 3$ cells were transfected with psgMstn1, psgMstn2 and psgMstn3 plasmids. The resulting genomic DNA was subjected to sequencing analysis. Sequencing revealed few off-target sites in the mouse genome, a result consistent with our previous observations in mouse cells. Six potential offtarget sites were identified for sgMstn1, five for sgMstn2 and another nine for sgMstn3, with a low probability of off-target sites in the mouse genome (Fig. 5A). We concluded that SaCas9 editing is intrinsically specific. We also performed targeted deep-sequencing with genomic DNA from the muscles of mice to validate these off-target sites. According to this more sensitive readout, indels detected more accurately above background at all of the offtarget locus 1 sites with Mstn (Fig. 5B). These results indicated that rAAV9-sgMstn1 is a promising and safe candidate for in vivo applications.

\section{Discussion}

Several CRISPR/Cas9-based-inducible systems have been developed, but few have been developed for the SaCas 9 variant. Since the characterization of SaCas9 [16], several groups have extended its application in targeted mutagenesis in a variety of models such as plants, mice and zebrafish[37-39]. Because of its smaller size, SaCas 9 provides substantial advantages in the delivery and expression of Cas9, especially when AAVs are used. Recent work has shown that SaCas9 has higher activity than the other Cas9 variants such as SpCas9 and FnCpf1[40].

To our knowledge, there are currently few available single AAV viruses containing both the Cas 9 and sgRNA. Among them, the SaCas 9 system has been shown to be effective for gene editing in AAV in vivo[16]. These systems not only provide comparable editing levels to those of other SpCas9 systems, but also have the advantage of being within the AAV packaging limit. Moreover, researchers can manipulate gene expression at precise sites and to precise degrees of expression. Therefore, these SaCas 9 systems can probably be used to edit genes at specific sites to improve tissue traits and treat chronic diseases[41, 42]. First, we report the development of an SaCas 9 system with a different sgRNA promoter (tRNA pro 72 bp) and SaCas 9 promoter (EF1a pro $212 \mathrm{bp}$ ) that can be delivered to cells via rAAV. This single vector system contains a smaller Cas9, a fluorescent protein and a gRNA expression cassette. Only one virus is needed to mediate genome editing in animals or cell lines. We believe that this system should be extremely useful and may be used separately or in combination with other expression cassettes in experiments.

We observed favorable gene editing efficiency by AAV in vitro across our experiments. Through infection of C2C12 cells, we observed fluorescence expression of the eGFP protein in rAAV-DJ/8-sgMstn1. One study has indicated that when the SaCas9 system targets the HT1080 cell line in vitro, the genome editing rate is $\sim 40-50 \%[43]$. In contrast, our system showed relatively low editing efficiency $\sim 17.31 \%$ in vitro with the same amount of virus, but by increasing the amount of virus, we substantially improved the editing efficiency approximately $\sim 24.84 \%$. We suspect that the recombinant system maybe slightly less efficient at editing in vitro, owing to lower sgRNA expression from the U6 to tRNA promoters. This possibility seems reasonable, given that the success of system depends on maintaining tight levels of promoter control of sgRNA expression. We found that increasing the amount of rAAV-DJ/8-sgMstn1 improved the expression of eGFP protein and the efficiency of SaCas 9 protein editing. We also found that rAAVSaCas 9 was expressed stably in cells for a long time in our experiments. Therefore, genome editing efficiency can be improved by increasing the expression time and titer of recombinant viruses.

Because of differences in AAV serotypes, transcription factors and injection sites, AAV-mediated delivery of transgenes in tissues often differs [29]. In addition, the titer of the AAV virus and the method of virus injection also greatly affect the efficiency of AAV-mediated transgene delivery. Intramuscular injection was used because it has a highly targeted effect on tissues. The effect is more easily analyzed by comparing the differences in muscle tissues, cells and corresponding proteins without considering the overall effect on local areas. In addition, systemic injection may cause adverse effects specific to nerves, immune cells, hormones and other factors, and may cause a large area of off-target editing and even cancer mutations in offspring. Mstn knockout in mice also resulted in changes in body characteristics. Therefore, we focused on local targeted knockout experiments in gene editing. In our in vivo experiments, we infused our rAAV-SaCas 9 system into the left thigh in C57BL/10 male mice. Therefore, genome editing was restricted to the SaCas 9 system within this region. We found that the higher the rAAV9-sgMstn1 titer, the higher the fluorescence intensity of eGFP protein; moreover, we were able to clearly observed the location of the rAAV9-sgMstn 1 through eGFP protein fluorescence. We observed the feasibility and effectiveness of genome editing in these systems, which achieved approximately $\sim 13.11 \%$ genome editing at 8 weeks. These editing efficiencies are higher than those in previous studies from laboratory examining SaCas 9 genome editing[23, 44]. In agreement with the results of in vitro experiments, increasing the amount of recombinant virus in vivo also promoted the genome editing efficiency of the virus. The genome editing efficiency in the high virus titer group was $17.59 \%$ at 8 weeks, and the greatest amount of genomic editing was $\sim 21.68 \%$. Therefore, within a certain period of time, when the amount and expression time of the virus increased, the gene editing of the virus in vivo achieved the best effect by using rAAV9-Mstn1.

Muscle wasting occurs with aging and in a wide range of catabolic diseases, such as cancer, diabetes, chronic renal disease and heart failure, which can dramatically decrease quality of life and increase disease mortality[23]. Recently, in vivo genome editing targeting muscle tissues with CRISPR-Cas systems has shown efficacy in a mouse model of Duchenne muscular dystrophy[45]. With an aim to establish a therapeutic treatment for muscle-

Page $7 / 15$ 
wasting syndrome, we used CRISPR/Cas9 to directly target Mstn in vivo in skeletal muscle cells to prevent loss of muscle mass. We believed that by blocking the MSTN protein expression and myostatin pathway of some skeletal muscle cells, these cells should be able to prevent the atrophy of target cells and adjacent cells, thus maintaining muscle function to a certain extent[46]. Our constructed rAAV system has been shown to be effective for gene editing of thigh muscles. Our AAV-SaCas9 viruses target muscle, owing to the natural tropism of AAV9 for muscle within the mouse leg. We immunostained the SaCas9 protein to indicate its expression in muscle and possible editing in vivo. Thomas and Joulia et al. have found that MSTN up-regulates the activity and level of cyclin-dependent kinase inhibitors, thus preventing $\mathrm{G} 1$ phase to $\mathrm{S}$ phase transition, inhibiting the proliferation of muscle cells, and decreasing the number of muscle fibers[47, 48]. McPherron and coworkers have found that mutant mice have significantly enlarged muscles; in mice with gene knockout of Mstn, the number of muscle fibers was $86 \%$ greater than that in wild-type mice, thus indicating that Mstn knockout causes muscle fiber growth and hypertrophy[19]. The results of our experiment are consistent with that conclusion. Treatment with rAAV9Mstn1 resulted in significantly higher body weight, quadriceps weight and adductor weight than those in the muscular dystrophy group. Through analysis of muscle tissue, we confirmed that partial knockout the Mstn gene improved the volume and number of muscle fibers, an effect particularly important for the treatment of muscular atrophy.

\section{Conclusion}

Gene editing for all of the systems could probably be optimized further by using highly efficient sgRNAs and high titer AAVs, increasing the incubation time of these viruses in vivo, and using serotypes of AAV that transduce target cells with high efficiency. In this paper, we demonstrate that the constructed rAAV-SaCas9 genome editing system confer stable expression in vitro and in vivo. Moreover, The fluorescence intensity of eGFP protein and the gene editing efficiency of SaCas 9 protein increased with increasing rAAV-SaCas 9 titer. The fluorescence intensity of the eGFP protein was also used to determine the infection effects and the location of the rAAV-SaCas9. In addition, we edited muscle-atrophic mice with a high dose of rAAV9SaCas 9 virus, which effectively improved the muscle properties of mice. The results show that the rAAV-SaCas 9 system has substantial therapeutic potential for muscular atrophy.

\section{Declarations}

\section{Acknowledgements}

Not applicable

\section{Funding}

This work was supported by grants from the Ministry of Agriculture and Rural Affairs of China (2016ZX08006001-006) and Advantage and Characteristic Discipline of Henan Province (203/18xk0102).

\section{Availability of data and materials}

Not applicable.

\section{Author Contributions}

Guoyu Yang, Jiang Wang and Shaoting Weng conceived and designed the experiments; Shaoting Weng, Xingyu Li, Mengmeng Shi, Haoxiang Yang and Feng Gao performed the cell studies; Shaoting Weng, Liqiang Han and Yitian Zhao performed the mouse studies; Shaoting Weng, Xingyu Li, Yitian Zhao and Liqiang Han analyzed the datas from the cell and mouse studies; Shaoting Weng wrote the manuscript; Guoyu Yang and Jiang Wang reviewed and edited the manuscript. All authors have read and agreed to the published version of the manuscript.

\section{Ethics approval and consent to participate}

Animal experiments were approved by the Animal Research Committee of Henan Agricultural University and all animals were maintained in a specific pathogen-free animal facility according to the related ethical regulations instilled at Henan Agricultural University and the guide for the care and use of laboratory animals.

\section{Consent for publication}


Not applicable.

\section{Competing interests}

The authors declare no competing interests.

\section{References}

1. Stella S, Montoya G: The genome editing revolution: A CRISPR-Cas TALE off-target story. Bioessays 2016, 38 Suppl 1:S4-s13.

2. Jiang DJ, Xu CL, Tsang SH: Revolution in Gene Medicine Therapy and Genome Surgery. Genes (Basel) $2018,9$.

3. Wan H, Feng C, Teng F, Yang S, Hu B, Niu Y, Xiang AP, Fang W, Ji W, Li W, et al: One-step generation of p53 gene biallelic mutant Cynomolgus monkey via the CRISPR/Cas system. Cell Res 2015, 25:258-261.

4. Yang H, Wang H, Shivalila CS, Cheng AW, Shi L, Jaenisch R: One-step generation of mice carrying reporter and conditional alleles by CRISPR/Casmediated genome engineering. Cell 2013, 154:1370-1379.

5. Xu L, Wang J, Liu Y, Xie L, Su B, Mou D, Wang L, Liu T, Wang X, Zhang B, et al: CRISPR-Edited Stem Cells in a Patient with HIV and Acute Lymphocytic Leukemia. 2019, 381:1240-1247.

6. Zhu S, Zhou Y, Wei W: Genome-Wide CRISPR/Cas9 Screening for High-Throughput Functional Genomics in Human Cells. Methods Mol Biol 2017, 1656:175-181.

7. Chen S, Sanjana NE, Zheng K, Shalem O, Lee K, Shi X, Scott DA, Song J, Pan JQ, Weissleder R, et al: Genome-wide CRISPR screen in a mouse model of tumor growth and metastasis. Cell 2015, 160:1246-1260.

8. Yang Y, Wang L, Bell P, McMenamin D, He Z, White J, Yu H, Xu C, Morizono H, Musunuru K, et al: A dual AAV system enables the Cas9-mediated correction of a metabolic liver disease in newborn mice. Nat Biotechnol 2016, 34:334-338.

9. Ekman FK, Ojala DS, Adil MM, Lopez PA, Schaffer DV, Gaj T: CRISPR-Cas9-Mediated Genome Editing Increases Lifespan and Improves Motor Deficits in a Huntington's Disease Mouse Model. Mol Ther Nucleic Acids 2019, 17:829-839.

10. Yu W, Wu Z: Use of AAV Vectors for CRISPR-Mediated In Vivo Genome Editing in the Retina. Methods Mol Biol 2019, 1950:123-139.

11. Moreno AM, Fu X, Zhu J, Katrekar D, Shih YV, Marlett J, Cabotaje J, Tat J, Naughton J, Lisowski L, et al: In Situ Gene Therapy via AAV-CRISPR-Cas9Mediated Targeted Gene Regulation. Mol Ther 2018, 26:1818-1827.

12. Crudele JM, Chamberlain JS: AAV-based gene therapies for the muscular dystrophies. Hum Mol Genet 2019, 28:R102-r107.

13. Tabebordbar M, Zhu K, Cheng JKW, Chew WL, Widrick JJ, Yan WX, Maesner C, Wu EY, Xiao R, Ran FA, et al: In vivo gene editing in dystrophic mouse muscle and muscle stem cells. Science 2016, 351:407-411.

14. Kleinstiver BP, Prew MS, Tsai SQ, Topkar VV, Nguyen NT, Zheng Z, Gonzales AP, Li Z, Peterson RT, Yeh JR, et al: Engineered CRISPR-Cas9 nucleases with altered PAM specificities. Nature 2015, 523:481-485.

15. Esvelt KM, Mali P, Braff JL, Moosburner M, Yaung SJ, Church GM: Orthogonal Cas9 proteins for RNA-guided gene regulation and editing. Nat Methods 2013, 10:1116-1121.

16. Ran FA, Cong L, Yan WX, Scott DA, Gootenberg JS, Kriz AJ, Zetsche B, Shalem O, Wu X, Makarova KS, et al: In vivo genome editing using Staphylococcus aureus Cas9. Nature 2015, 520:186-191.

17. Mefferd AL, Kornepati AV, Bogerd HP, Kennedy EM, Cullen BR: Expression of CRISPR/Cas single guide RNAs using small tRNA promoters. Rna 2015, 21:1683-1689.

18. Zincarelli C, Soltys S, Rengo G, Rabinowitz JE: Analysis of AAV serotypes 1-9 mediated gene expression and tropism in mice after systemic injection. Mol Ther 2008, 16:1073-1080.

19. McPherron AC, Lawler AM, Lee SJ: Regulation of skeletal muscle mass in mice by a new TGF-beta superfamily member. Nature 1997, $387: 83-90$.

20. McPherron AC, Lee SJ: Double muscling in cattle due to mutations in the myostatin gene. Proc Natl Acad Sci U S A 1997, 94:12457-12461.

21. Camporez JP, Petersen MC, Abudukadier A, Moreira GV, Jurczak MJ, Friedman G, Haqq CM, Petersen KF, Shulman Gl: Anti-myostatin antibody increases muscle mass and strength and improves insulin sensitivity in old mice. Proc Natl Acad Sci U S A 2016, 113:2212-2217.

22. Smith RC, Lin BK: Myostatin inhibitors as therapies for muscle wasting associated with cancer and other disorders. Curr Opin Support Palliat Care 2013, 7:352-360.

23. Wei Y, Chen Y, Qiu Y, Zhao H, Liu G, Zhang Y, Meng Q, Wu G, Chen Y, Cai X, et al: Prevention of Muscle Wasting by CRISPR/Cas9-mediated Disruption of Myostatin In Vivo. Mol Ther 2016, 24:1889-1891.

24. Gallot YS, Durieux AC, Castells J, Desgeorges MM, Vernus B, Plantureux L, Rémond D, Jahnke VE, Lefai E, Dardevet D, et al: Myostatin gene inactivation prevents skeletal muscle wasting in cancer. Cancer Res 2014, 74:7344-7356.

25. Weng S, Gao F, Wang J, Li X, Chu B, Wang J, Yang G: Improvement of muscular atrophy by AAV-SaCas9-mediated myostatin gene editing in aged mice. Cancer Gene Ther 2020. 
26. Cotton TR, Fischer G: Structure of the human myostatin precursor and determinants of growth factor latency. 2018, 37:367-383.

27. Rodriguez J, Vernus B, Toubiana M, Jublanc E, Tintignac L, Leibovitch S, Bonnieu A: Myostatin inactivation increases myotube size through regulation of translational initiation machinery. J Cell Biochem 2011, 112:3531-3542.

28. Salzler RR, Shah D, Doré A, Bauerlein R, Miloscio L, Latres E, Papadopoulos NJ, Olson WC, MacDonald D, Duan X: Myostatin deficiency but not antimyostatin blockade induces marked proteomic changes in mouse skeletal muscle. Proteomics 2016, 16:2019-2027.

29. Senis E, Fatouros C, Grosse S, Wiedtke E, Niopek D, Mueller AK, Borner K, Grimm D: CRISPR/Cas9-mediated genome engineering: an adenoassociated viral (AAV) vector toolbox. Biotechnol J 2014, 9:1402-1412.

30. Huang X, Zhou G, Wu W, Duan Y, Ma G, Song J, Xiao R, Vandenberghe L, Zhang F, D'Amore PA, Lei H: Genome editing abrogates angiogenesis in vivo. Nat Commun 2017, 8:112.

31. Holehonnur R, Luong JA, Chaturvedi D, Ho A, Lella SK, Hosek MP, Ploski JE: Adeno-associated viral serotypes produce differing titers and differentially transduce neurons within the rat basal and lateral amygdala. BMC Neurosci 2014, 15:28.

32. Macedo AG, Krug AL, Souza LM, Martuscelli AM, Constantino PB, Zago AS, Rush JW, Santos CF, Amaral SL: Time-course changes of catabolic proteins following muscle atrophy induced by dexamethasone. Steroids 2016, 107:30-36.

33. Feldman AT, Wolfe D: Tissue processing and hematoxylin and eosin staining. Methods Mol Biol 2014, 1180:31-43.

34. Schaaf GJ, van Gestel TJ, Brusse E, Verdijk RM, de Coo IF, van Doorn PA, van der Ploeg AT, Pijnappel WW: Lack of robust satellite cell activation and muscle regeneration during the progression of Pompe disease. Acta Neuropathol Commun 2015, 3:65.

35. Xu L, Lau YS, Gao Y, Li H, Han R: Life-Long AAV-Mediated CRISPR Genome Editing in Dystrophic Heart Improves Cardiomyopathy without Causing Serious Lesions in mdx Mice. Mol Ther 2019.

36. Ohmori T, Nagao Y, Mizukami H, Sakata A, Muramatsu SI: CRISPR/Cas9-mediated genome editing via postnatal administration of AAV vector cures haemophilia B mice. 2017, 7:4159.

37. Kaya H, Ishibashi K, Toki S: A Split Staphylococcus aureus Cas9 as a Compact Genome-Editing Tool in Plants. Plant Cell Physiol 2017, 58:643649.

38. Xu L, Gao Y, Lau YS, Han R: Adeno-Associated Virus-Mediated Delivery of CRISPR for Cardiac Gene Editing in Mice. J Vis Exp 2018.

39. Feng Y, Chen C, Han Y, Chen Z, Lu X, Liang F, Li S, Qin W, Lin S: Expanding CRISPR/Cas9 Genome Editing Capacity in Zebrafish Using SaCas9. G3 (Bethesda) 2016, 6:2517-2521.

40. Xie H, Tang L, He X, Liu X, Zhou C, Liu J, Ge X, Li J, Liu C, Zhao J, et al: SaCas9 Requires 5'-NNGRRT-3' PAM for Sufficient Cleavage and Possesses Higher Cleavage Activity than SpCas9 or FnCpf1 in Human Cells. Biotechnol J 2018, 13:e1700561.

41. De Caneva A, Porro F, Bortolussi G, Sola R, Lisjak M, Barzel A, Giacca M, Kay MA, Vlahovicek K, Zentilin L, Muro AF: Coupling AAV-mediated promoterless gene targeting to SaCas9 nuclease to efficiently correct liver metabolic diseases. JCI Insight $2019,5$.

42. Xu L, Lau YS, Gao Y, Li H, Han R: Life-Long AAV-Mediated CRISPR Genome Editing in Dystrophic Heart Improves Cardiomyopathy without Causing Serious Lesions in mdx Mice. Mol Ther 2019, 27:1407-1414.

43. Morsy SG, Tonne JM, Zhu Y, Lu B, Budzik K, Krempski JW, Ali SA, El-Feky MA, Ikeda Y: Divergent susceptibilities to AAV-SaCas9-gRNA vectormediated genome-editing in a single-cell-derived cell population. BMC Res Notes 2017, 10:720.

44. Kumar N, Stanford W, de Solis C, Aradhana, Abraham ND, Dao TJ, Thaseen S, Sairavi A, Gonzalez CU, Ploski JE: The Development of an AAV-Based CRISPR SaCas9 Genome Editing System That Can Be Delivered to Neurons in vivo and Regulated via Doxycycline and Cre-Recombinase. Front Mol Neurosci 2018, 11:413.

45. Lim KRQ, Yoon C, Yokota T: Applications of CRISPR/Cas9 for the Treatment of Duchenne Muscular Dystrophy. $2018,8$.

46. Cohen S, Nathan JA, Goldberg AL: Muscle wasting in disease: molecular mechanisms and promising therapies. Nature reviews Drug discovery $2015,14: 58-74$.

47. Thomas M, Langley B, Berry C, Sharma M, Kirk S, Bass J, Kambadur R: Myostatin, a negative regulator of muscle growth, functions by inhibiting myoblast proliferation. J Biol Chem 2000, 275:40235-40243.

48. Joulia D, Bernardi H, Garandel V, Rabenoelina F, Vernus B, Cabello G: Mechanisms involved in the inhibition of myoblast proliferation and differentiation by myostatin. Exp Cell Res 2003, 286:263-275.

\section{Figures}


1.

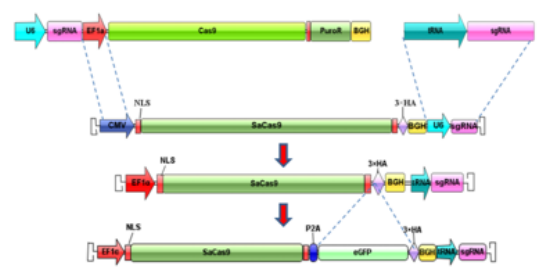

IB

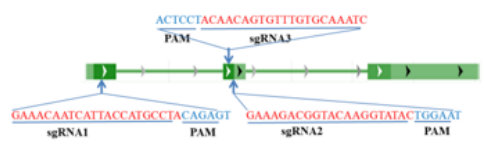

1C

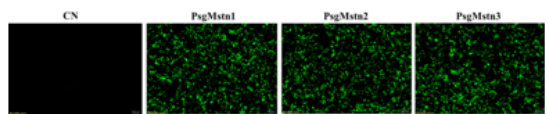

ID|
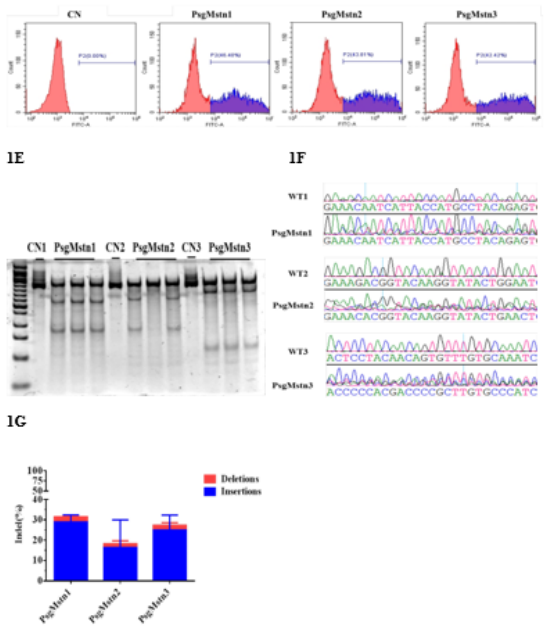

Figure 1

The SaCas9/CRISPR system exhibits efficient genome editing in vitro. (A) AAV vector maps depicting EF1a:SaCas9-eGFP-tRNA:sgRNA. EF1a:SaCas9eGFP-tRNA:sgRNA is an AAV vector that consists of an EF1a promoter controlling the expression of the coding regions of SaCas9, followed by a selfcleaving P2A peptide sequence and then an eGFP Flag. The sgRNA expression cassette is under the control of an tRNA promoter. (B) The approximate location of the sgRNA binding site in the Mstn locus. These three target sites were all located in the conserved N-terminal sequence in the first and second exons of the Mstn gene, which play an important role in MSTN protein synthesis. The sgRNAs, or target sequences, are underlined, and the PAM sequence (NNGRRT) is specified. (C) pX601-EF1a:SaCas9-eGFP-tRNA:sgRNA plasmid was transfected into NIH/3T3 cells. For the control group (CN), similar cells were transfected with pX601. After $72 \mathrm{~h}$, eGFP fluorescence was observed to determine eGFP expression. (D) The eGFP fluorescence expression of transfected cells was calculated by flow cytometry. The abscissa represents the value of the relative strength of the eGFP fluorescence signal, the ordinate is the number of cells, and P2 shows the percentage of eGFP fluorescent cells relative to the total number of cells. (E) The treatment groups of NIH/3T3 cells were transfected with psgMstn1/psgMstn2/psgMstn3. The control group of NIH/3T3 cells was transfected with pX601. At 72 $\mathrm{h}$ post transfection, cells were harvested, and genomic DNA was isolated and purified. The $521 \mathrm{bp}, 548 \mathrm{bp}$ and $537 \mathrm{bp}$ regions of the sgMstn1/sgMstn2/sgMstn3 locus, including the site targeted for editing, were PCR amplified from genomic DNA and analyzed for genome editing with a T7 endonuclease digestion identification method. The top band on the gel is uncut DNA at 521 bp, 548 bp and 537 bp in length, and the two smaller bands are edited DNA at 181 and 340 bp in length for sgMstn1, 185 and 352 bp in length for sgMstn2, and 122 and 415 bp in length for sgMstn3. The nucleic acid marker was a 50 bp DNA ladder. (F) The sgMstn1/sgMstn2/sgMstn3 knockout sequence peak was obtained by Sanger sequencing. For a control, genomic DNA of the pX601-treated group was sequenced. (G) Stacked histogram showing the percentage distribution of indels at sgMstn1, sgMstn2 and sgMstn3 in NIH/3T3 mutant cells, as measured by sequencing analyses. Data represent means \pm SD from three technical replicates. 


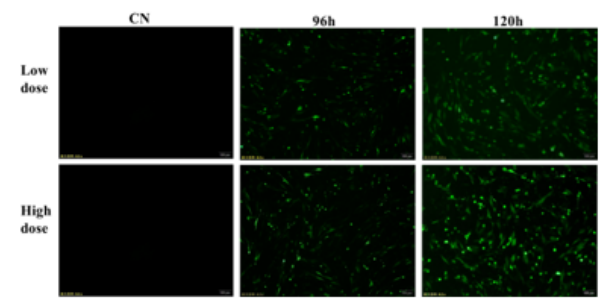

2B

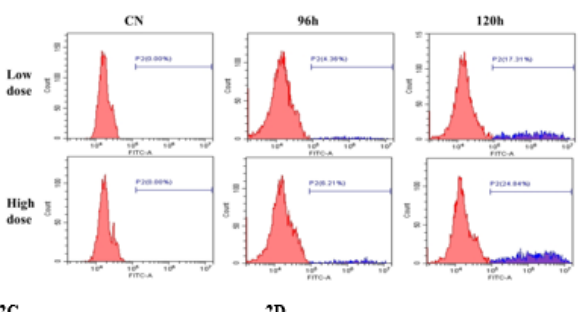

2C

2D
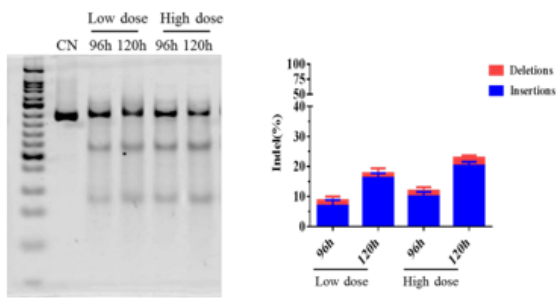

$2 \mathrm{E}$

2F
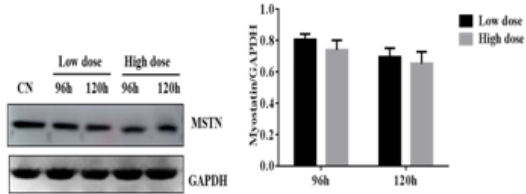

Figure 2

The rAAV-DJ/8-SaCas9 system efficiently targets the Mstn site in C2C12 cells. (A) Low/high dose rAAV-DJ/8-sgMstn1 infected into C2C12 cells. For the control group, similar cells were infected with rAAV-DJ/8-CN. At 96 or $120 \mathrm{~h}$ later, eGFP fluorescence was observed to determine eGFP expression. (B) The eGFP fluorescence expression of infected cells was calculated by flow cytometry. The abscissa represents the value of the relative strength of the eGFP fluorescence signal, the ordinate is the number of cells, and P2 shows the percentage of eGFP fluorescent cells relative to the total number of cells. (C) C2C12 cells were infected with low/high dose AAV-DJ/8-sgMstn1. At 96 and at $120 \mathrm{~h}$ post infection, cells were harvested, and genomic DNA was isolated and purified. A 521 bp region of the sgMstn1 locus including the site targeted for editing was PCR amplified from the genomic DNA and analyzed for genome editing with a T7 endonuclease digestion identification method. The top band on the gel is uncut DNA at 521 bp in length, and the two smaller bands are edited DNA at 181 and $340 \mathrm{bp}$ in length for sgMstn 1 at 96 and $120 \mathrm{~h}$ post infection. The nucleic acid marker was a 50 bp DNA ladder. (D) Stacked histogram showing the percentage distribution of indels at sgMstn1 in C2C12 mutant cells, as measured by sequencing analyses. Data represent means \pm SD from three technical replicates. (E) The two dose rAAV-DJ/8-sgMstn1 treated C2C12 cells, analyzed by western blotting with an anti-MSTN antibody at 96 and $120 \mathrm{~h}$. Anti-GAPDH was used as a loading control. (F) Stacked histogram showing the percentage distribution of MSTN expression in different C2C12 cells. The MSTN value of each group was divided by the corresponding GAPDH value, and then the data for each group were divided by the same multiple to normalize by the control group value. Data represent means \pm SD from three technical replicates. 

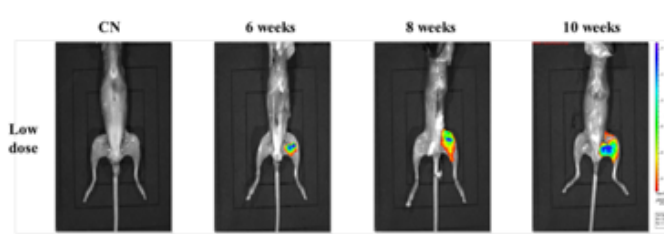

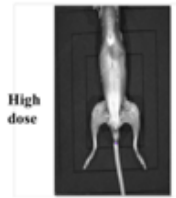

$3 \mathrm{~B}$
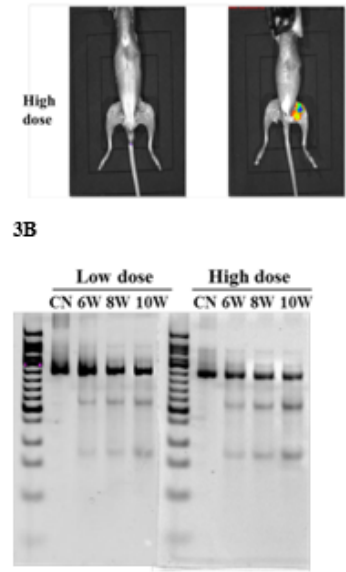

3D
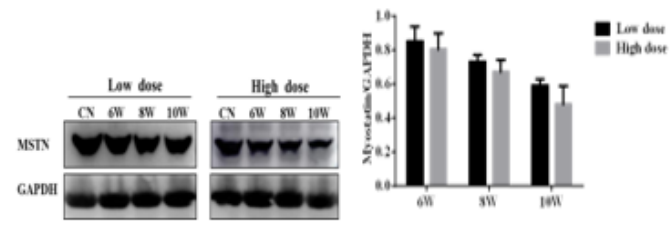

Figure 3

The rAAV9-SaCas9 system efficiently targets the Mstn site in C57BL/10 male mice thigh muscle. (A) For the rAAV9-SaCas9 system, the expression range was $2 \times 1010-5 \times 1010$ photons/second/cm 2. The images are shown at 6, 8 and 10 weeks post-injection (dpi). Owing to the weak fluorescence expression of eGFP, fluorescence imaging results were not visible through the mouse fur. Therefore, we killed the mice first and then stripped off the fur for fluorescence imaging. To exclude rAAV9-CN expression in the thigh, a control group is included in the first image for the low/high dose rAAV9sgMstn1 treatment groups (ventral position). (B) Mouse muscles were infected with low/high rAAV9-sgMstn1 in the treatment groups, and the control groups were infected with the same dose of rAAV9-CN. At 6, 8 and 10 weeks (with) post infection, mouse muscle was harvested, and genomic DNA was isolated and purified. A $521 \mathrm{bp}$ region of the sgMstn1 locus including the site targeted for editing was PCR amplified from the genomic DNA and analyzed for genome editing with a T7 endonuclease digestion identification method. The top band on the gel is uncut DNA at 521 bp in length in all groups, but the two smaller bands are edited DNA at 181 and 340 bp in length for sgMstn 1 at 6, 8 and 10 weeks only in the treatment groups. The nucleic acid marker was a 50 bp DNA ladder. (C) Stacked histogram showing the percentage distribution of indels at sgMstn1 in thigh muscle in mice with low/high dose rAAV9-sgMstn1, as measured by sequencing analyses. Data represent means \pm SD from three technical replicates. (D) Mouse left thigh muscles were treated with low/high dose rAAV9-sgMstn1 and rAAV9-CN, analyzed by western blotting with anti-MSTN antibody at 6, 8 and 10 weeks post infection. Anti-GAPDH was used as a loading control. (E) Stacked histogram showing the percentage distribution of MSTN expression in different mouse thigh muscles treated with low/high dose rAAV9-sgMstn1 and rAAV9-CN. The MSTN value of each group was divided by the corresponding GAPDH value, and then the data of each group were divided by the same multiple to normalize by the control group value. Data represent means \pm SD from three technical replicates. 


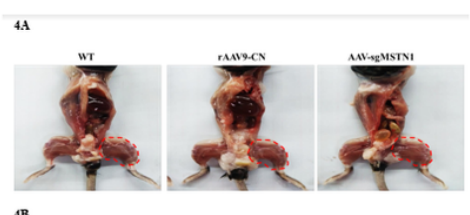

$4 \mathrm{~B}$

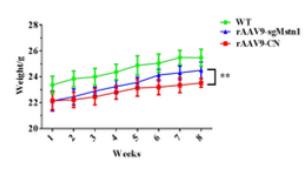

4D
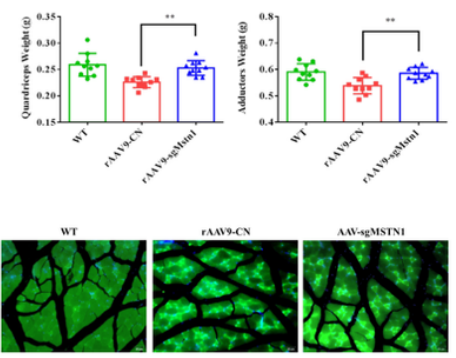

$4 \mathrm{~F}$

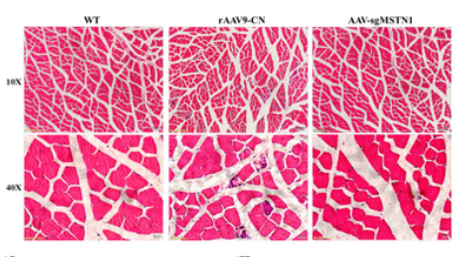

46

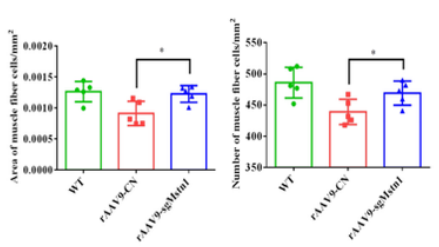

Figure 4

The rAAV9-SaCas9 system efficiently improves muscle mass in muscle-atrophic mice. (A) Changes in left thigh muscle mass in C57BL/10 male mice in the WT, rAAV9-CN treated and rAAV9-sgMstn1-treated groups (ventral position). Compared with that in the rAAV9-CN treated group, the muscle mass in the rAAV9-sgMstn1-treated group was significantly increased (red cycle). (B) Changes in body weight from 1 to 8 weeks in different experimental groups. (C) and (D) The weights of the quadriceps and adductor muscle in the different groups at 8 weeks. N=10/group, *: $0.01<P<0.05 \nabla^{\star *}$ : $P<0.01$ for the rAAV9-sgMstn1-treated group compared with the WT and rAAV9-CN treated groups. (E) Tissue immunofluorescence tests were performed on different groups of mouse muscle sections (scale bar $=50 \mu \mathrm{m}$ ). FITC fluorescence was observed for SaCas 9 expression. Images depict mouse leg muscle examined for SaCas9 expression. Similar fields of view also depict DAPI stained nuclei. (F) Sections were cut from each quadriceps muscle and stained with hematoxylin and eosin (H \& E) to visually determine the myofiber shape. Representative images of H\&E staining of WT, rAAV9-CN treated and rAAV9-sgMstn1-treated muscle sections are shown (scale bar $=100 \mu \mathrm{m} \otimes 20 \mu \mathrm{m})$. (G) The mean value of muscle fiber areas (mm2) as well as $(H)$ the mean value of muscle fiber density within each section was measured. $N=5$ /group, $*$ : $0.01<P<0.05 \nabla^{\star *}$ : $P<0.01$ for the rAAV9-sgMstn 1 treated group compared with the WT and rAAV9-CN treated groups. 
$5 A$

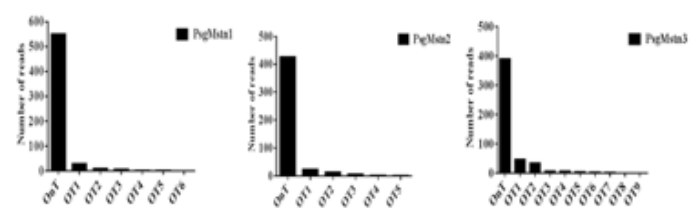

$5 B$

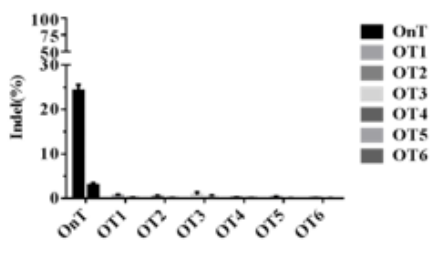

Figure 5

SaCas9 is highly specific in vivo. (A) Numbers of sequencing reads for the on-target (OnT) and off-target (OT) sites of sgMstn1, sgMstn2 and sgMstn3 in $\mathrm{C} 2 \mathrm{C} 12$ cells. (B) Targeted deep-sequencing to measure the muscle tissues at each OT site of sgMstn1. 